\title{
Diagnoseirrtum versus Befunderhebungsfehler - Ein Update der Rechtsprechung mit Fallbeispielen
}

\section{Einleitung}

Bereits der RöFo-Beitrag 08/2017 „Irren ist menschlich: Haftung wegen Diagnose- und/ oder Befunderhebungsfehlern sowie Aufklärungsfehlern im Rahmen des MammografieScreenings" setzte sich mit dem Diagnoseirrtum, dessen Voraussetzungen und der Abgrenzung zu dem - haftungsrechtlich gesehen - weit schwerwiegenderen Befunderhebungsfehler sowie möglichen Aufklärungsfehlern auseinander. Daran anknüpfend greift der vorliegende Beitrag die aktuelle Rechtsprechung zu diesem Themenkreis auf, die sich mit den Unterschieden zwischen den beiden Fehlertypen weiter auseinandersetzt. Im Rahmen eines Arzthaftungsprozesses ist von entscheidender Bedeutung, ob ein vermeintlicher ärztlicher Fehler als nicht vorwerfbarer Diagnoseirrtum, als Diagnosefehler oder aber als Befunderhebungsfehler eingeordnet wird. Nicht zuletzt erfolgt diese Einschätzung des jeweiligen Gerichts mithilfe von medizinischen Sachverständigen, denen als naturgemäß - juristische Laien die Unterschiede nicht immer vollends bekannt sind. Der Diagnoseirrtum ist nur sehr restriktiv überhaupt als Behandlungsfehler anzuerkennen, wohingegen es bei einem Befunderhebungsfehler sogar dann zu einer sonst nur bei groben Behandlungsfehlern auftretenden Beweislastumkehr zwischen dem Behandlungsfehler und dem Primärschaden zugunsten des Patienten kommen kann, wenn der Fehler an sich nur als einfacher Behandlungsfehler eingestuft wird, sich bei der gebotenen Befunderhebung jedoch ein Befund gezeigt hätte, auf den sich eine Nichtreaktion als grob fehlerhaft darstellen würde. Die Kenntnis über die Unterschiede der Fehlertypen ist damit auch für die Ärzteschaft hilfreich.

\section{Definition Diagnoseirrtum und Diagnosefehler}

Ein nicht als Behandlungsfehler zu wertender „bloßer“ Diagnoseirrtum liegt vor, wenn der Behandelnde erhobene oder sonst vorliegende Befunde falsch interpretiert und deshalb nicht die aus der berufsfachlichen Sicht seines Fachbereichs gebotenen - therapeutischen oder diagnostischen - Maßnahmen ergreift (vgl. BGH Urt. v. 21.01.2016, Az.: VI ZR 146/14). Die Diagnose ist dabei der Schluss von den erhobenen Befunden auf eine konkrete Erkrankung. Zugrunde zu legen sind alle verfügbaren Befunde. Auch vor Zufallsbefunden, die nicht hätten erhoben werden müssen, darf der Arzt die Augen nicht verschließen (vgl. BGH Urt.v. 21.12.2010, Az.: VI ZR 284/09).

Dagegen liegt ein vorwerfbarer Diagnosefehler nur dann vor, wenn die richtige Diagnose grob fehlerhaft verkannt oder eine Verdachts- bzw. Arbeitsdiagnose nicht überprüft wurde und die infolgedessen gestellte Diagnose aus fachärztlicher Sicht nicht mehr vertretbar erscheint.

Liegt also ein fundamentaler Diagnoseirrtum vor, ist regelmäßig von einem groben Behandlungsfehler auszugehen. Jenseits solcher eindeutiger Diagnosefehler bleibt nur dann Raum für eine Haftung des Arztes, wenn die von ihm erhobenen Befunde nicht zweifelhaft sind, sondern bei Anwendung der gebotenen Sorgfalt nur den Schluss auf eine bestimmte Diagnose zulassen (vgl. OLG Dresden, Urt. v. 29.07.2019, Az.: 4 U 1078/19; OLG Köln, VersR 2004, 794).

\section{Abgrenzung zum Befunderhebungsfehler}

Diagnoseirrtum und Diagnosefehler setzen daher voraus, dass der Behandler die medizinisch gebotenen Befunde überhaupt erhoben hat, um sich eine ausreichende Basis für die Einordnung der Krankheitssymptome zu verschaffen.

Hat dagegen die unrichtige diagnostische Einstufung einer Erkrankung ihren Grund bereits darin, dass der Behandler die nach dem medizinischen Stand gebotenen Befunde erst gar nicht veranlasst hat, liegt ein Befunderhebungsfehler vor (vgl. BGH, Urt. v. 26.01.2016, Az.: VI ZR 146/14).

Zwingende Voraussetzung für die Annahme eines Befunderhebungsfehlers ist demnach,

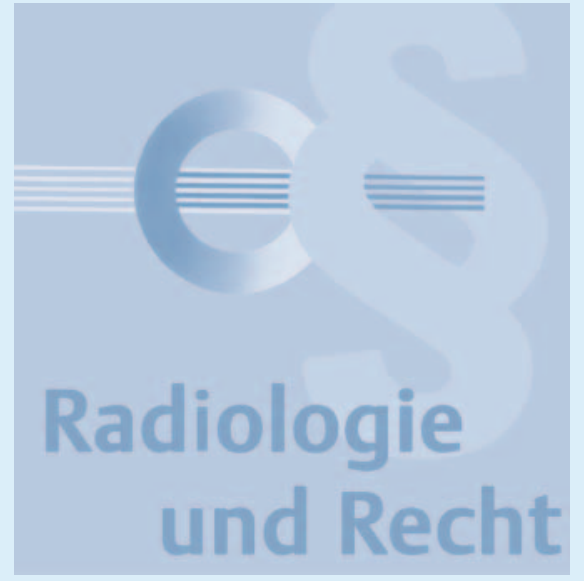

dass die Erhebung medizinisch gebotener Befunde unterlassen wurde, sodass auch die Frage, in welchem Umfang und aus wessen fachärztlicher Sicht Befunde erhoben werden müssen, bedeutend ist.

\section{Update Rechtsprechung}

Die Gerichte haben sich immer wieder damit zu befassen, welche Befunde aus fachärztlicher Sicht hätten erhoben werden müssen und ob eine aufgrund der vorliegenden Befunde gestellte Diagnose noch vertretbar erscheint. Dabei stehen bei den Landgerichten spezielle Arzthaftungskammern zur Verfügung, welche mit 3 Richtern besetzt sind. In unproblematischen Fällen kann das Gericht auch durch die/den Einzelrichter/in entscheiden. Von Vorteil ist bei den Land- und Oberlandesgerichten, dass die dortigen Richter in den Fachkammern über ein gutes Basiswissen zu medizinischen Sachverhalten und ein Spezialwissen zu den medizinrechtlichen Feinheiten verfügen.

Ein kurzer Überblick über die aktuelle Rechtsprechung soll die Abgrenzungskriterien verdeutlichen, anhand derer die Gerichte die Fehlertypen einordnen.

\section{Brandenburgisches Oberlandes- gericht, Urteil vom 25.04.2019}

Mit Urteil vom 25.04.2019 hat das Brandenburgische Oberlandesgericht die Berufung einer Patientin gegen ein erstinstanzlich teilweise klageabweisendes Urteil zurückgewiesen und eine Verurteilung der beklagten Klinik zur Zahlung von $5000 €$ Schmerzensgeld und die Feststellung der Ersatzpflicht für künftige materielle und 
immaterielle Schäden bestätigt. In dieser Entscheidung wurden zum einen ein nicht vorwerfbarer Diagnoseirrtum und zum anderen ein Befunderhebungsfehler angenommen.

Die Ärzte der beklagten Klinik waren zunächst aufgrund geklagter linksseitiger Schmerzen der Patientin von einer Obstipation ausgegangen, was sich im Nachhinein als fehlerhaft herausstellte. Tatsächlich hatte die Patientin einen Niereninfarkt erlitten. Da die Beklagte die erforderlichen Befunde, nämlich Anamnese, Sonografie und Röntgen, klinische Untersuchung und das Überprüfen für einen Niereninfarkt typischer Symptome durchgeführt, beide Nieren für klinisch ohne pathologischen Befund beurteilt und eine Darmproblematik festgestellt hatte, sei die fehlerhafte Diagnose jedoch nicht unvertretbar und damit nicht vorwerfbar gewesen. Anlass für weitere bildgebende Verfahren oder die Ermittlung des LDH-Wertes habe nicht bestanden.

Einen Befunderhebungsfehler sah das Gericht jedoch darin, dass am Folgetag keine CT-Untersuchung und eine Gabe von Heparin-Bolus als sofortige Antikoagulation sowie eine zu geringe Medikation mit Clexane durchgeführt worden seien. Denn nach anhaltenden Schmerzen, trotz Stuhlgangs und erhöhter Schmerzmedikation, habe die Arbeitsdiagnose Obstipation nicht mehr aufrechterhalten werden können und eine weitere Befunderhebung sei angezeigt gewesen. Im CT hätte sodann derjenige Befund erkannt werden können, welcher bereits am Vortag vorgelegen habe, nämlich ein Nierenarterienverschluss. Dieser hätte zu einem sofortigen intravenösen HeparinBolus mit 5000-10000 IE und niedermolekularem Heparin von 2-mal 70 mg täglich für mehrere Tage führen müssen. Der Klägerin wurden jedoch lediglich 1 -mal $20 \mathrm{mg}$ für 3 Tage, 1 -mal $40 \mathrm{mg}$ für 4 Tage sowie 2-mal 40 mg täglich zum Ende der Behandlung gegeben. Die unterlassene HeparinBolus-Gabe und die folgende Unterdosierung seien nicht mehr nachvollziehbar, sodass es sich hierbei um einen groben Behandlungsfehler handele, welcher zu einer Beweislastumkehr zulasten des beklagten Klinikums führte.

Eine vollständige Wiederherstellung der Nierenfunktion sei jedoch auch bei einer sofortigen Behandlung de lege artis nicht mehr zu erreichen gewesen, weswegen lediglich zulasten der beklagten Klinik gewertet wurde, dass sich nicht mehr aufklären ließ, inwieweit bei richtiger Behandlung eine Besserung der Nierenfunktion hätte erreicht werden können. Somit wurde bei der Bemessung des Schmerzensgeldes nicht die schicksalhafte Nierenerkrankung an sich, sondern das aufgrund der Zeitverzögerung mitverursachte Risiko einer Dialysepflicht berücksichtigt, woraus der relativ geringe Schmerzensgeldbetrag i. H.v. $5000 €$ resultiert.

In dieser Entscheidung zeigt sich sehr deutlich, dass eine eingangs gestellte Diagnose aufgrund hinzutretender oder veränderter Symptome stetig überprüft werden muss notfalls durch weitere Befunderhebungen.

\section{Oberlandesgericht Köln, Urteil vom 09.01.2019}

Die Pflichten für eine vollständige Befunderhebung können im Einzelfall auch gesteigert sein. So verhielt es sich in einem dem Urteil des Oberlandesgerichts Köln vom 09.01.2019, Az.: I 5 U 13/17 zugrunde liegenden Sachverhalt.

Der Patient stellte sich nach einem Arbeitsunfall, bei dem er nach einem Sprung vom LKW mit dem Fuß umgeknickt war, im Krankenhaus vor. Der Durchgangsarzt veranlasste eine Röntgenaufnahme des oberen Sprunggelenks (OSG) und diagnostizierte eine Verstauchung und Zerrung des OSG sowie Spontanruptur nicht näher bezeichneter Sehnen. Er verordnete einen Kompressionsverband, eine Aircast-Schiene sowie Kühlung und übernahm die weitere Behandlung. 9 Tage später vermerkte er in seiner Dokumentation u. a.: „Minimale Schwellung, weiterhin keine Schmerzen (diabetische Polyneuropathie)“. Zwischenzeitlich wurde der Kläger auch von seinem Hausarzt und in 2 anderen Krankenhäusern behandelt, u. a. wurde die Ausbildung eines Charcot-Fußes bei Vorliegen einer proximalen Mittelfußfraktur diagnostiziert.

Der Kläger hat behauptet, er habe im Rahmen des Arbeitsunfalls eine Basisfraktur am fünften Mittelfußknochen davongetragen mit der Konsequenz, dass die Knochenenden zunehmend auseinandergewichen seien und eine lokale Zirkulationsstörung aufgetreten sei. Diese habe zu der katastrophalen Entwicklung der Ausbildung eines Charcot-Fußes geführt. Infolge dessen könne der Kläger seinen linken Fuß nicht mehr einsetzen und das linke Bein nur noch wie eine Stelze verwenden. Sowohl der Durchgangsarzt, als Beklagter zu 1, als auch der Hausarzt, als Beklagter zu 2, der über den Hergang des Arbeitsunfalls informiert worden sei, hätten es unterlassen, die erforderliche Befunderhebung durchzuführen.

Das OLG Köln entschied, dass der Beklagte zu 1 es vorwerfbar unterlassen habe, spätestens als ihm die diabetische Grunderkrankung und eine darauf beruhende Polyneuropathie bekannt waren, die zuvor gestellte Diagnose eines reinen Umknicktraumas des linken Fußes mit Verstauchung und Zerrung im Sprunggelenk sowie einer Spontanruptur nicht näher bezeichneter Sehnen kritisch zu hinterfragen, eine mögliche Mitbeteiligung des 5. Mittelfußknochens in Erwägung zu ziehen und eine entsprechend erweiterte Diagnostik durch Röntgenaufnahmen des Fußes zu veranlassen. Jedenfalls mit der Kenntnis dieser Sensibilitätsstörung und der persistierenden Beschwerden musste der Beklagte zu 1 die Möglichkeit einer übersehenen Fraktur im Fuß bedenken und vor dem Hintergrund der erheblichen Komplikationsgefahren, die aus einer solchen übersehenen Fraktur resultierten, unverzüglich handeln.

Das Versäumnis weiterer röntgenologischer Abklärung stelle sich keineswegs als bloßer Diagnoseirrtum dar, der seinerseits nicht als Behandlungsfehler anzusehen wäre, sondern als Befunderhebungsmangel. Der Kern des Vorwurfs läge nicht in einer möglicherweise unrichtigen Erstdiagnose, sondern in dem Versäumen, diese durch notwendige weitergehende Diagnostik abzusichern. Es gehe um die Nichterhebung zweifelsfrei gebotener Befunde.

Die geforderte Röntgenuntersuchung hätte aus Sicht des Gerichts das Ergebnis einer Fraktur erbracht, sodass zwischen der unterlassenen Befunderhebung und dem Gesundheitsschaden des Klägers eine Kausalität bestehe. Denn ein derartiger Befund hätte zwingend weitergehende therapeutische Reaktionen erfordert, die zu unterlassen sich als grober Behandlungsfehler 
dargestellt hätte. Ein solches Versäumnis stellt einen Verstoß gegen fachärztlichen Standard und damit eine Pflichtverletzung dar.

Wegen des ausgebildeten Charcot-Fußes wurde dem Kläger ein Schmerzensgeld i. H. v. $50000 €$ zugesprochen.

Aufgrund der hinzugetretenen Kenntnis der Diabetes mit einer Polyneuropathie hätte der Durchgangsarzt demnach aus Sicht des Gerichts die zunächst gestellte Diagnose kritisch hinterfragen und eine Fraktur röntgenologisch ausschließen müssen.

\section{Landgericht Flensburg, Urteil vom 02.08.2019}

Mit der Frage, welcher Facharztstandard bei der Erkennbarkeit einer richtigen Diagnose und zur Beurteilung der Notwendigkeit zu erhebender Befunde zugrunde zu legen ist, hatte sich das Landgericht Flensburg in seinem Urteil vom 02.08.2019, Az.: 3 O 198/15 auseinanderzusetzen.

Die Durchführung und Befundung einer Röntgenaufnahme des Thorax gehöre zumindest auch (noch) in das Fachgebiet eines niedergelassenen Pneumologen. Bei der Durchführung und ausschließlichen Befundung einer Röntgenaufnahme des Thorax hat ein niedergelassener Facharzt für Innere Medizin und Pneumologie deshalb den Standard seines Fachgebiets und nicht den eines Radiologen zu wahren.

Grundlage der Entscheidung war eine lungenärztliche Behandlung eines Patienten, bei dem ein Bronchialkarzinom verkannt worden war. Der Patient verstarb während des Verfahrens. Die Anamnese hatte einen seit zwei Monaten bestehenden Reizhusten, Schmerzen im Bereich des Brustkorbs seit ca. einem Monat, Abgeschlagenheit, Antriebslosigkeit sowie auffällige Atemgeräusche im Liegen ergeben und der Patient berichtete von einem vorausgegangenen unspezifischen Infekt nach einem Aufenthalt in der Eishalle.

Ein durchgeführter Lungenfunktionstest war unauffällig, ebenfalls ein von der Beklagten durchgeführter bronchialer Provokationstest. Die Beklagte führte sodann eine Röntgenuntersuchung des Thorax durch. Hierzu heißt es in der Behandlungsdokumentation: „Rö 2 EB 31.1.2014 opB.“ In dem Arztbrief der Beklagten hieß es u. a.: „Unauffälliger Befund an Lunge, Herz, Pleura und Mediastinum. Kein Hinweis auf Pneumonie, kein Anhalt für Tumor.“ Und schließlich: „PROC WV wenn Beschwerden länger als 4 Wochen anhaltend, dann Thorax-CT ...". Ob dies mit dem Patienten auch so besprochen wurde, war zwischen den Parteien streitig.

Vier Monate später stellte sich der Patient erneut vor und klagte über persistierende Schmerzen im Burstkorb, woraufhin nach klinischer Untersuchung und Lungenfunktionstest die Diagnose Asthma bronchiale gestellt wurde. Erneut wurde vermerkt: „Wenn in 2 Wochen nicht besser, dann kurzfristig CT-Thorax ... PROC WV 3m, wenn nicht besser, dann kurzfristig CT-Thorax."

Etwa vier Wochen später wurde der Patient stationär aufgenommen und im Rahmen einer Röntgenuntersuchung ein „... Verplumpter Hilus links sowie Plattenatelektase im linken Mittelfeld“ festgestellt. Der Befund sollte zum Ausschluss einer zentralen Raumforderung computertomografisch abgeklärt werden. Ein kontrastmittelgestütztes CT wurde schließlich durchgeführt. Bei dem Patienten wurde ein Lungenkarzinom links zentral mit der „Tumorformel: cT4 N0/2 Mx (entspricht Stadium III A/B) ... Adenokarzinom" diagnostiziert.

Das Landgericht lehnte eine Haftung der erstbehandelnden Pneumologin ab, da ein Diagnosefehler nicht vorliege. Zwar zeigte sich bei der ex post durchgeführten Nachbefundung des ersten Röntgenbildes durch den im Verfahren bestellten Sachverständigen bereits eine links-hiläre tumoröse Raumforderung. Diese hatte die Pneumologin bei der Auswertung des Röntgenbildes verkannt. Eine solche Fehlinterpretation erhobener Befunde (sog. „Diagnoseirrtum“) werde in der Rechtsprechung aber nur zurückhaltend als Behandlungsfehler (sog. „Diagnosefehler") bewertet: Ein Behandlungsfehler sei nicht immer schon dann anzunehmen, wenn ein Arzt zu einer objektiv unrichtigen Diagnose gelange. Grundsätzlich sei zwar das Nichterkennen einer erkennbaren Erkrankung und der für sie kennzeichnenden Symptome als Behandlungsfehler zu werten. Irrtümer bei der
Diagnosestellung, die in der Praxis nicht selten vorkommen, seien jedoch oft nicht die Folge eines vorwerfbaren Versehens des Arztes. Die Symptome einer Erkrankung seien nämlich nicht immer eindeutig, sondern könnten auf die verschiedensten Ursachen hinweisen. Dies gelte auch unter Berücksichtigung der vielfachen technischen Hilfsmittel, die zur Gewinnung von zutreffenden Untersuchungsergebnissen einzusetzen seien. Auch könne jeder Patient wegen der Unterschiedlichkeiten des menschlichen Organismus die Anzeichen ein und derselben Krankheit in anderer Ausprägung aufweisen. Diagnoseirrtümer, die objektiv auf eine Fehlinterpretation der Befunde zurückzuführen seien, könnten deshalb nur mit Zurückhaltung als Behandlungsfehler gewertet werden (vgl. BGH, Urt. v. 08.07.2003, Az.: I ZR 304/02).

Dem stehe auch nicht entgegen, dass der von dem Sachverständigen zusätzlich herangezogene Radiologe in seinem schriftlichen Gutachten ausgeführt habe, die beschriebene Raumforderung hätte vermutet und in einem schriftlichen Befund Erwähnung finden müssen. Maßgeblicher ärztlicher Standard für die Beurteilung sei hier der eines Facharztes für Innere Medizin und Pneumologie, nicht derjenige eines Radiologen. Dieser Facharztstandard sei von der Pneumologin durch die gestellte, objektiv fehlerhafte Diagnose nicht in vorwerfbarer Weise unterschritten worden.

\section{Fazit}

Sobald die aus fachärztlicher Sicht erforderlichen Befunde vollständig erhoben worden sind, kann bereits ein Befunderhebungsfehler nicht mehr angenommen werden. Die Vollständigkeit der erhobenen Befunde richtet sich dabei nach einer Einzelfallbetrachtung und kann nicht für ein bestimmtes Krankheitsbild pauschaliert festgelegt werden. Ist es aufgrund der erhobenen und vorliegenden Befunde zu einer Fehleinschätzung des Befundes gekommen, so ist von einem Diagnoseirrtum auszugehen. Sofern die gestellte Diagnose fachärztlich vertretbar erscheint, ist ein solcher Irrtum nicht haftungsrechtlich vorwerfbar.

Grundsätzlich gilt, dass ein Patient ausgehend von seinem Erwartungshorizont be 
Aufsuchen eines Arztes Anspruch auf Einhaltung des Standards guter ärztlicher Versorgung nach Maßstab eines erfahrenen Arztes der jeweiligen Fachrichtung (sog. „Facharztstandard“) hat (Frahm/Walter, Arzthaftungsrecht, 6. Aufl., Rn. 9; OLG Schleswig, Urt. v. 24.06.2005, Az.: 4 U 10/ 04). Wenn es sich um einen Facharzt für Radiologie handelt, so ist dies grundsätzlich auch der maßgebliche Facharztstandard. Wendet ein Arzt allerdings Untersuchungsund Behandlungsmethoden an, die in ein fremdes Fachgebiet fallen, hat er dessen Standard zu garantieren (Frahm/Walter, $\mathrm{aaO}$, Rn. 86).

Gehört also die Durchführung und ausschließliche Befundung einer speziellen Röntgenaufnahme allein in das Fachgebiet der Radiologie, wäre demnach der maßgebliche Facharztstandard der eines Radiologen. Entsprechend hat das LG Flensburg mit Urt. v. 28.02.2019, Az.: 30 5/14 entschieden, dass für die Anfertigung und Beurteilung einer Mammografie auch ein Frauenarzt den Facharztstandard eines Radiologen einzuhalten hat. Ausgangspunkt für die Einordnung sind die Weiterbildungsinhalte der jeweiligen Facharztausbildung.
Die obergerichtliche Rechtsprechung geht zudem davon aus, dass demjenigen Arzt, welcher eine nicht vorwerfbar falsche Diagnose gestellt und deswegen - aus seiner Sicht folgerichtig - bestimmte Befunde nicht erhoben hat, aufgrund der unrichtigen Diagnosestellung keine unterlassene Befunderhebung vorzuwerfen sei (u. a. OLG München, Urt. v. 22.03.2012, Az.: 1 U 1244/11 \& Urt. v. 29.03.2012, Az.: 1 U 36/ 11 \& Urt. v. 06.10.2011, Az.: 1 U 5220/10; OLG Koblenz, Urt. v. 20.01.2011, Az.: 5 U $828 / 10)$.

Aus einem Diagnoseirrtum kann damit nicht unmittelbar ein Befunderhebungsfehler resultieren, da es sich ansonsten um einen Zirkelschluss handeln würde. Denn der Arzt geht bei dem Diagnoseirrtum schließlich von einer Diagnose aus, welche weitere Befunderhebungen aus seiner Sicht entbehrlich macht, sodass ihm die Unterlassung der weiteren Befunderhebung dann nicht wiederum als Behandlungsfehler vorgeworfen werden kann.

Dies gilt jedoch nur solange, wie sich die zum Zeitpunkt des Stellens der - irrtümlichen Diagnose erhobenen Befunde nicht verändert haben. Werden nachträglich erhebliche
Vorerkrankungen bekannt, verschlechtert sich der Gesundheitszustand eines Patienten oder treten neue bzw. veränderte Symptome auf, welche mit der irrtümlich gestellten Diagnose nicht in Einklang zu bringen sind, so ist von neuem zu prüfen, ob weitergehende Befunde aus fachärztlicher Sicht zu erheben sind oder nicht. Im Zweifel sollte hierbei immer der sicherste Weg gewählt werden, soweit dem Patienten unter Abwägung des Schaden-Nutzen-Risikos eine (weitere) Strahlenbelastung o. ä. im Rahmen neuer bzw. weiterer Befunderhebungsmaßnahmen zugemutet werden kann.

Prof. Dr. Peter Wigge

Rechtsanwalt

Fachanwalt für Medizinrecht

Christina Feldmeier-Budelmann

Rechtsanwältin

Fachanwältin für Medizinrecht

Rechtsanwälte Wigge

Großer Burstah 42

20457 Hamburg

Telefon: (040) 3398 705-90

Telefax: (040) 3398 705-99

E-Mail: hamburg@ra-wigge.de www.ra-wigge.de 